\title{
A NOTE ON LOCALISED WEIGHTED INEQUALITIES FOR THE EXTENSION OPERATOR
}

\author{
J. A. BARCELÓ, J. M. BENNETT ${ }^{\bowtie}$ and A. CARBERY
}

(Received 6 March 2006; accepted 12 February 2007)

Communicated by G. A. Willis

\begin{abstract}
We prove optimal radially weighted $L^{2}$-norm inequalities for the Fourier extension operator associated to the unit sphere in $\mathbb{R}^{n}$. Such inequalities valid at all scales are well understood. The purpose of this short paper is to establish certain more delicate single-scale versions of these.
\end{abstract}

2000 Mathematics subject classification: 42B10.

Keywords and phrases: Fourier extension operators, weighted inequalities.

\section{Introduction}

Let $n \geq 2$ and $\sigma$ denote the induced Lebesgue measure on the unit sphere $\mathbb{S}^{n-1} \subset \mathbb{R}^{n}$. We define the extension operator associated to $\mathbb{S}^{n-1}$ to be the mapping $g \mapsto \widehat{g d \sigma}$, where

$$
\widehat{g d \sigma}(x)=\int_{\mathbb{S}^{n-1}} g(\xi) e^{-2 \pi i x \cdot \xi} d \sigma(\xi),
$$

$g \in L^{1}\left(\mathbb{S}^{n-1}\right)$ and $x \in \mathbb{R}^{n}$. In this note we are concerned with the validity of certain weighted inequalities for this extension operator. The most well-known conjectured inequality of this type is due to Mizohata and Takeuchi; see [9]. In what follows $\mu$ will be a nonnegative Borel measure on the unit ball $\mathbb{B}$ of $\mathbb{R}^{n}$. For such a measure we define

$$
\|\mu\|:=\sup \frac{\mu(T)}{w(T)^{n-1}}
$$

where the sup is taken over all infinite rectangles $T$ in $\mathbb{R}^{n}$ with $(n-1)$ short sides with common length $w(T)$ and remaining side doubly infinite.

All authors were supported by the EC project 'HARP'. The first was also supported by Spanish Grant BFM02206, the second by EPSRC Postdoctoral Fellowship GR/S27009/02 and the third by a Leverhulme Study Abroad Fellowship and EC project 'Pythagoras'.

(c) 2008 Australian Mathematical Society 1446-7887/08 \$A2.00+0.00 
CONJECTURE 1.1. There exists a constant $C$, depending at most upon $n$, such that if the measure $\mu$ satisfies \|\|$\mu \| \mid<\infty$ and $R \geq 1$, then

$$
\int_{\mathbb{B}}|\widehat{g d \sigma}(R x)|^{2} d \mu \leq C \frac{\|\mu\| \mid}{R^{n-1}}\|g\|_{L^{2}\left(\mathbb{S}^{n-1}\right)}^{2}
$$

for all $g \in L^{2}\left(\mathbb{S}^{n-1}\right)$.

This conjecture has been verified in the case where the measure $\mu$ is radial; this is done in both [2] and [4]. For more general weights very little is known beyond some very special examples.

In this note we consider the versions of these inequalities which arise upon fixing the scale $R$ in Conjecture 1.1. (Both versions of this conjecture arise naturally in a number of problems on the interface between harmonic analysis and geometric measure theory; see, for example, [3-6, 13].) We now describe this set-up, beginning with some notation.

Let $R \geq 1$ be a (large) parameter which may now be considered fixed. For $R^{-1} \leq \alpha \leq R^{-1 / 2}$, let $T\left(\alpha, \alpha^{2} R\right)$ denote a rectangle (or 'tube') of arbitrary position and orientation in $\mathbb{R}^{n}$, having $n-1$ short sides of length $\alpha$ and one long side of length $\alpha^{2} R$; so at one extreme, $T\left(\alpha, \alpha^{2} R\right)$ is a cube of side $R^{-1}$, and at the other, $T\left(\alpha, \alpha^{2} R\right)$ is a rectangle of long side of length 1 and $n-1$ short sides of length $R^{-1 / 2}$.

We test our extension operator on the standard examples that generate the classical restriction conjecture for the Fourier transform (see [10]). One simply takes the function $g$ to be an arbitrary (modulated) characteristic function of a $\delta$-ball in $\mathbb{S}^{n-1}$; that is $g(\xi)=e^{i a \cdot \xi} \chi(\xi)$ where $a \in \mathbb{R}^{n}$, and $\chi$ is the characteristic function of a $\delta$-ball in $\mathbb{S}^{n-1}$ for some $0<\delta \leq 1$. The fact that $|\widehat{g d \sigma}(R x)|$ is large on a suitable tube suggests the possibility that the inequality

$$
\int_{\mathbb{B}}|\widehat{g d \sigma}(R x)|^{2} d \mu \leq \frac{C}{R^{n-1}} \sup _{R^{-1} \leq \alpha \leq R^{-1 / 2}}\left\{\frac{\mu\left(T\left(\alpha, \alpha^{2} R\right)\right)}{\alpha^{n-1}}\right\}\|g\|_{L^{2}\left(\mathbb{S}^{n-1}\right)}^{2},
$$

might hold for all $g \in L^{2}\left(\mathbb{S}^{n-1}\right)$. Here $C$ is a constant depending on at most $n$. Observe that this has the correct homogeneity in the sense that the multiscale inequality (1.1) would be a consequence of this on letting $R \rightarrow \infty$; the key point being that for any rectangle $T\left(\alpha, \alpha^{2} R\right)$,

$$
\frac{\mu\left(T\left(\alpha, \alpha^{2} R\right)\right)}{\alpha^{n-1}} \leq\|\| \mu \|
$$

uniformly in $\alpha$ and $R$.

This observation also shows that the condition $\|\mu\| \mid<\infty$ is necessary for an inequality of the form (1.1) to hold uniformly in $R$.

Unlike in the multi-scale situation, this single-scale inequality (1.2) turns out to be false even for radial $\mu$. Curiously, however, in the radial case, we are able to prove a slightly weaker statement which is, nonetheless, optimal (up to multiplicative constants). This is the content of our main theorem. 
THEOREM 1.2. Let $\mu$ be a non-negative radial Borel measure supported on $\mathbb{B}$. There exists a constant $0<C<\infty$, depending on at most $n$, such that

$$
\int_{\mathbb{B}}|\widehat{g d \sigma}(R x)|^{2} d \mu \leq C \frac{\log \log R}{R^{n-1}} \sup _{R^{-1} \leq \alpha \leq R^{-1 / 2}}\left\{\frac{\mu\left(T\left(\alpha, \alpha^{2} R\right)\right)}{\alpha^{n-1}}\right\}\|g\|_{L^{2}\left(\mathbb{S}^{n-1}\right)}^{2}
$$

for all $g \in L^{2}\left(\mathbb{S}^{n-1}\right)$ and $R \geq 1$. Conversely, there exists a constant $0<c<\infty$, depending on at most $n$, such that for each $R \geq 1$, there is a nonnegative radial Borel measure $\mu$ supported on $\mathbb{B}$ for which

$$
\int_{\mathbb{B}}|\widehat{d \sigma}(R x)|^{2} d \mu \geq c \frac{\log \log R}{R^{n-1}} \sup _{R^{-1} \leq \alpha \leq R^{-1 / 2}}\left\{\frac{\mu\left(T\left(\alpha, \alpha^{2} R\right)\right)}{\alpha^{n-1}}\right\} .
$$

It should be remarked that although inequality (1.3) is sharp, it just fails to possess the correct homogeneity to imply the corresponding multiscale inequality (inequality (1.1) for $\mu$ radial).

Although the main result concerns radial measures, its proof contains some inequalities for general measures which are perhaps of interest. We mention these below as a corollary.

If we instead consider the same problem where the sphere is replaced by the base of a paraboloid, then the analogue of (1.2) fails by a power of $R$ rather than just by a factor of $\log \log R$. See [1]. See also [7] for some further results.

In the next section we establish some preliminary lemmas, and in Section 3 we give a proof of Theorem 1.2, and some further remarks concerning its formulation. Finally in Section 4 we make some remarks on known variants of inequality (1.2) for general (that is nonradial) measures $\mu$ in two dimensions.

Notation For nonnegative quantities $X$ and $Y$ we use $X \lesssim Y(X \gtrsim Y)$ to denote the existence of a positive constant $C$, depending on at most $n$, such that $X \leq C Y$ $(X \geq C Y)$. We write $X \sim Y$ if both $X \lesssim Y$ and $X \gtrsim Y$.

\section{Lemmas}

As we shall see, since our measures $\mu$ are radial, we may quickly reduce the proof of Theorem 1.2 to certain uniform estimates for ordinary Bessel functions. The following lemma makes a connection between the relevant Bessel function asymptotics and expressions of a geometric nature involving tubes. Here we use $d \mathcal{H}^{n-2}$ to denote the $n-2$ dimensional Hausdorff measure on $\mathbb{R}^{n}$.

LEMMA 2.1. Let $0<\alpha \leq \beta, r>0$ and $u, \omega \in \mathbb{S}^{n-1}$ with $u \cdot \omega=0$. We define $a$ rectangle $T=T_{r, \omega, u}(\alpha, \beta) \subset \mathbb{R}^{n}$ as follows:

(1) $T$ has $n-1$ short sides of length $\alpha$ and one long side of length $\beta$;

(2) $T$ is centred at the point $r \omega$, and has direction $u$. 
If

$$
\beta^{2}>8 r \alpha+4 \alpha^{2}
$$

and $x \in \mathbb{R}^{n}$ is such that

$$
r+\alpha \leq|x| \leq \sqrt{r^{2}+\frac{\beta^{2}}{4}},
$$

then the quantity

$$
I_{n}(x, r, \alpha, \beta):=\int_{\mathbb{S}^{n-1}} \int_{\left\{u \in \mathbb{S}^{n-1}: u \cdot \omega=0\right\}} \chi_{T_{r, \omega, u}(\alpha, \beta)}(x) d \mathcal{H}^{n-2}(u) d \sigma(\omega)
$$

satisfies

$$
I_{n}(x, r, \alpha, \beta) \sim \frac{\alpha^{n-1}}{|x|^{n-3 / 2}(|x|-r)^{1 / 2}} .
$$

PROOF. We begin by observing that $I_{n}(x, r, \alpha, \beta)$ is a radial function of $x$, and that the integral

$$
\int_{\mathbb{S}^{n-1}} \chi_{T_{r, \omega, u}(\alpha, \beta)}(t|x|) d \sigma(t)
$$

is independent of $\omega$ and $u$. Using these observations and Fubini's theorem, we may write

$$
\begin{aligned}
I_{n}(x, r, \alpha, \beta)= & \frac{1}{c_{n-1}} \int_{\mathbb{S}^{n-1}} \int_{\mathbb{S}^{n-1}} \int_{\left\{u \in \mathbb{S}^{n-1}: u \cdot \omega=0\right\}} \chi_{T_{r, \omega, u}\left(\alpha, \alpha^{2} R\right)}(t|x|) \\
& \times d \mathcal{H}^{n-2}(u) d \sigma(\omega) d \sigma(t) \\
= & \frac{1}{c_{n-1}} \int_{\mathbb{S}^{n-1}} \chi_{T_{r, \omega^{\prime}, u^{\prime}}\left(\alpha, \alpha^{2} R\right)}(t|x|) d \sigma(t),
\end{aligned}
$$

where $c_{n-1}$ denotes the volume of the unit sphere in $\mathbb{R}^{n}$, and $\omega^{\prime}$ and $u^{\prime}$ are arbitrary points on $\mathbb{S}^{n-1}$ with $u^{\prime} \cdot \omega^{\prime}=0$. For convenience we choose $\omega^{\prime}=(0,0, \ldots, 1)$ and $u^{\prime}=(1,0, \ldots, 0)$.

Now fix $x \in \mathbb{R}^{n}$ and set $\rho=|x|$. By (2.1) we have that

$$
\sqrt{r^{2}+\frac{\beta^{2}}{4}}>r+\alpha
$$

To obtain (2.3) in the region (2.2), we must calculate the proportion of $\rho \mathbb{S}^{n-1}$ that intersects $T_{r, \omega^{\prime}, u^{\prime}}(\alpha, \beta)$. This intersection is an 'ellipsoid' in $\rho \mathbb{S}^{n-1}$ with length $|P-Q|$ in one direction and $\alpha$ in the remaining $n-2$ directions. Here

$$
P=\left(\sqrt{\rho^{2}-(r+\alpha)^{2}}, 0, \ldots, 0, r+\alpha\right) \text { and } Q=\left(\sqrt{\rho^{2}-r^{2}}, 0, \ldots, 0, r\right) .
$$

Hence

$$
I_{n}(x, r, \alpha, \beta) \sim \frac{|P-Q| \alpha^{n-2}}{\rho^{n-1}},
$$


and since

$$
\begin{aligned}
|P-Q|^{2} & \sim \alpha^{2}+\left(\sqrt{\rho^{2}-r^{2}}-\sqrt{\rho^{2}-(r+\alpha)^{2}}\right)^{2} \\
& \sim \alpha^{2}\left(1+\frac{(2 r+\alpha)^{2}}{\rho^{2}-r^{2}}\right) \\
& \sim \frac{\alpha^{2} \rho}{\rho-r}
\end{aligned}
$$

we obtain (2.3).

The following lemma summarises the relevant Bessel function estimates. See, for example, [2] for a proof.

LEMMA 2.2. For $v \geq 0$, there exists a universal constant $C>0$ such that

(1) for $0<r \leq 1$,

$$
\left|J_{v}(r)\right| \leq C\left(\frac{r}{2}\right)^{v} \frac{1}{\Gamma(v+1)}
$$

(2) for $r \geq 1$,

$$
\left|J_{v}(r)\right| \leq C r^{-1 / 2} \min \left\{v^{1 / 6},\left|\frac{r+v}{r-v}\right|^{1 / 4}\right\} .
$$

Incidentally, Lemma 2.2 fails to reflect a certain exponential decay of $J_{v}(r)$ present in the region $1 \leq r \leq v$ (see, for example, [12] for explicit details of this). Given this, it is perhaps of little surprise that we only need to use our geometric representation (given by Lemma 2.1) of these asymptotic estimates for $J_{v}(r)$ in the region $r \geq v$.

\section{The proof of Theorem $\mathbf{1 . 2}$}

We begin by constructing an example showing that our inequality is optimal.

Let $\mu$ be a radially nonincreasing Borel measure supported in $\mathbb{B}$. Observe that the supremum in the right-hand side of (1.2) is attained by a rectangle, centred at the origin, and whose long side is parallel to the $x_{1}$-axis. For this rectangle $T=T\left(\alpha, \alpha^{2} R\right)$ we have that

$$
\frac{1}{\alpha^{n-1}} \mu\left(T\left(\alpha, \alpha^{2} R\right)\right) \lesssim \frac{1}{\alpha} \int_{0}^{\alpha} t d \mu(t)+\int_{\alpha}^{\alpha^{2} R} d \mu(t) .
$$

Next we define a sequence of positive real numbers $\left\{\alpha_{j}\right\}_{j=0}^{k}$ by setting $\alpha_{0}=0$, $\alpha_{1}=2 / R$ and

$$
\alpha_{j+1}=R \alpha_{j}^{2} \quad \text { for } 2 \leq j<k,
$$

where $k$ is such that $\alpha_{k} \sim R^{-1 / 2}$. We now define the measure $\mu$ by

$$
d \mu(t)=\sum_{j=0}^{k} \frac{1}{\alpha_{j+1}} \chi_{\left(\alpha_{j}, \alpha_{j+1}\right]}(t) d t
$$


Observe that since $\alpha_{j}=2^{2^{j-1}} / R$, we have that

$$
k \sim \log \log R
$$

Let $g \equiv 1$. Using the well-known asymptotic formula (see [10])

$$
\widehat{d \sigma}(x)=c|x|^{-(n-1) / 2} \cos (|x|-\pi / 4)+O\left(|x|^{-(n+1) / 2}\right) \quad \text { as }|x| \rightarrow \infty,
$$

and the nature of the measure $\mu$, we obtain the lower bound

$$
\int|\widehat{d \sigma}(R x)|^{2} d \mu(x) \gtrsim \frac{1}{R^{n-1}} \int_{1 / R \leq|x| \leq 1} \frac{1}{|x|^{n-1}} d \mu(x) \gtrsim \frac{\log \log R}{R^{n-1}} .
$$

This gives the desired inequality since by (3.1),

$$
\sup _{R^{-1} \leq \alpha \leq R^{-1 / 2}}\left\{\frac{\mu\left(T\left(\alpha, \alpha^{2} R\right)\right)}{\alpha^{n-1}}\right\} \lesssim 1 .
$$

In order to prove the positive part of Theorem 1.2 we decompose $g$ into its spherical harmonics, writing

$$
g=\sum_{\ell} \sum_{m=1}^{a_{\ell}} c_{\ell, m} Y_{m}^{\ell}
$$

where $Y_{m}^{\ell}$ denotes the $m$ th standard basis element of the spherical harmonics of order $\ell$. By a standard orthogonality argument it is enough to show that

$$
\mathcal{J}:=\int_{|x|<1} \frac{\left|J_{v}(R x)\right|^{2}}{|R x|^{n-2}} d \mu(x) \lesssim \frac{\log \log R}{R^{n-1}}\|\mu\|_{R}
$$

where

$$
\|\mu\|_{R}:=\sup _{R^{-1} \leq \alpha \leq R^{-1 / 2}}\left\{\frac{\mu\left(T\left(\alpha, \alpha^{2} R\right)\right)}{\alpha^{n-1}}\right\}
$$

and $v=v(\ell)=\ell+(n-2) / 2$. To this end we break up $\mathcal{J}$ according to the behaviour of the Bessel function $J_{v}$ (see Lemma 2.2), writing $\mathcal{J}=\mathcal{J}_{1}+\mathcal{J}_{2}+\mathcal{J}_{3}+\mathcal{J}_{4}+\mathcal{J}_{5}$, where

$$
\begin{gathered}
\mathcal{J}_{1}=\int_{|R x| \leq 1} \frac{\left|J_{v}(R x)\right|^{2}}{|R x|^{n-2}} d \mu(x), \\
\mathcal{J}_{2}=\int_{1 \leq|R x| \leq \frac{v}{2},|x| \leq 1} \frac{\left|J_{v}(R x)\right|^{2}}{|R x|^{n-2}} d \mu(x), \\
\mathcal{J}_{3}=\int_{\nu / 2 \leq|R x| \leq v-2 v^{1 / 3},|x| \leq 1} \frac{\left|J_{v}(R x)\right|^{2}}{|R x|^{n-2}} d \mu(x), \\
\mathcal{J}_{4}=\int_{\nu-2 v^{1 / 3} \leq|R x| \leq v+2 v^{1 / 3},|x| \leq 1} \frac{\left|J_{v}(R x)\right|^{2}}{|R x|^{n-2}} d \mu(x)
\end{gathered}
$$


and

$$
\mathcal{J}_{5}=\int_{|R x| \geq v+2 v^{1 / 3},|x| \leq 1} \frac{\left|J_{v}(R x)\right|^{2}}{|R x|^{n-2}} d \mu(x) .
$$

We now obtain the required bounds for each of the $\mathcal{J}_{i}$ with $1 \leq i \leq 5$.

From our geometric point of view, the most interesting and significant term here is $\mathcal{J}_{5}$ (see the remark after the statement of Lemma 2.2). Although the arguments for the remaining terms are relatively straightforward, we include them for completeness.

By Lemma 2.2 and the fact that $v \geq(n-2) / 2$,

$$
\mathcal{J}_{1} \leq \mu\left(B\left(0, R^{-1}\right)\right) \lesssim \frac{1}{R^{n-1}}\|\mu\|_{R}
$$

Here we are using the fact that if $\alpha=R^{-1}$, then $T\left(\alpha, \alpha^{2} R\right)$ is simply a ball of radius $R^{-1}$.

By a straightforward dyadic decomposition, and Lemma 2.2 again, we have that

$$
\mathcal{J}_{2} \lesssim \frac{1}{v} \sum_{1 \leq 2^{k} \leq v / 2} \frac{1}{2^{k(n-2)}} \mu\left(B\left(0, R^{-1} 2^{k}\right)\right)
$$

Covering the ball $B\left(0, R^{-1} 2^{k}\right)$ with a union of (essentially disjoint) tubes of the form $T=T\left(\alpha, \alpha^{2} R\right)$, where $\alpha=2^{k / 2} / R$, of which there are a total of $O\left(2^{k / 2(n-2)}\right)$, quickly leads to the required bound.

We now turn to $\mathcal{J}_{4}$, and cover the annulus

$$
\Omega=\left\{x \in \mathbb{R}^{n}: \frac{v}{R}-\frac{2 v^{1 / 3}}{R} \leq|x| \leq \frac{v}{R}+\frac{2 v^{1 / 3}}{R}\right\}
$$

with $O\left(v^{(2 / 3) n-1}\right)$ tubes $T=T\left(\alpha, \alpha^{2} R\right)$, where $\alpha \sim v^{1 / 3} / R$, having bounded overlap. Thus, by Lemma 2.2,

$$
\mathcal{J}_{4} \lesssim \frac{1}{v^{n-(4 / 3)}} \int_{\Omega} d \mu(x) \lesssim \frac{1}{v^{n-(4 / 3)}} \sum_{T} \int_{T\left(\alpha, \alpha^{2} R\right)} d \mu(x) \lesssim \frac{1}{R^{n-1}}\|\mu\|_{R} .
$$

In order to bound $\mathcal{J}_{3}$ we let

$$
\Omega_{j}:=\left\{x \in \mathbb{R}^{n}: \frac{v}{R}-2^{j+1} \frac{v^{1 / 3}}{R} \leq|x| \leq \frac{v}{R}-2^{j} \frac{v^{1 / 3}}{R}\right\},
$$

and, using Lemma 2.2, write

$$
\mathcal{J}_{3} \lesssim \sum_{1 \leq 2^{j} \lesssim \nu^{2 / 3}} \frac{1}{R^{n-2} \nu^{n-4 / 3} 2^{j / 2}} \int_{\Omega_{j}} d \mu(x)
$$

and continue as in the case of $\mathcal{J}_{4}$, where for each $j$ the corresponding value of $\alpha$ is $2^{j} v^{1 / 3} R^{-1}$. 
We now turn to $\mathcal{J}_{5}$, where by Lemma 2.2

$$
\mathcal{J}_{5} \lesssim \frac{1}{R^{n-1}} \int_{\nu / R+2 v^{1 / 3} / R \leq|x| \leq 1} \frac{1}{|x|^{n-3 / 2}|| x|-v / R|^{1 / 2}} d \mu(x) .
$$

In order to bound this term we define a sequence $\left\{\alpha_{j}\right\}$ by $\alpha_{1}=2 \nu^{1 / 3} / R$ and

$$
\alpha_{j+1}=\frac{\alpha_{j}^{4} R^{2}}{2 r}=\frac{2^{1 / 3+2 / 34^{j}} v^{1 / 3}}{R}, \quad j \geq 1
$$

Let $k$ be such that $v / R+\alpha_{k} \sim R^{-1 / 2}$. As before we note that

$$
k \sim \log \log R
$$

We now write

$$
\mathcal{J}_{5} \lesssim \frac{1}{R^{n-1}} \sum_{j=1}^{k} \int_{\nu / R+\alpha_{j} \leq|x| \leq \nu / R+\alpha_{j+1}} \frac{1}{|x|^{n-3 / 2}|| x|-v / R|^{1 / 2}} d \mu(x),
$$

and apply Lemma 2.2 for each $j$, with $r=v / R, \alpha=\alpha_{j}$ and $\beta / 2=\alpha_{j}^{2} R$, to obtain

$$
\begin{aligned}
\mathcal{J}_{5} \lesssim & \frac{1}{R^{n-1}} \sum_{j=1}^{k} \frac{1}{\alpha_{j}^{n-1}} \int_{\nu / R+\alpha_{j} \leq|x| \leq \nu / R+\alpha_{j+1}} \int_{\mathbb{S}^{n-1}} \int_{\left\{u \in \mathbb{S}^{n-1}: u \cdot \omega=0\right\}} \\
& \times \chi_{T_{\nu / R, \omega, u}\left(\alpha_{j}, \alpha_{j}^{2} R\right)}(x) d \mathcal{H}^{n-2}(u) d \sigma(\omega) d \mu(x) \\
\lesssim & \frac{1}{R^{n-1}} \sum_{j=1}^{k} \int_{\mathbb{S}^{n-1}} \int_{\left\{u \in \mathbb{S}^{n-1}: u \cdot \omega=0\right\}} \frac{\mu\left(T_{\nu / R, \omega, u}\left(\alpha_{j}, \alpha_{j}^{2} R\right)\right)}{\alpha_{j}^{n-1}} d \mathcal{H}^{n-2}(u) d \sigma(\omega) \\
\lesssim & \frac{\log \log R}{R^{n-1}}\|\mu\|_{R},
\end{aligned}
$$

as required.

COROLlary 3.1. For all Borel measures $\mu$ supported in $\mathbb{B}$,

$$
\int_{|x|<1} \frac{\left|J_{v}(R x)\right|^{2}}{|R x|^{n-2}} d \mu(x) \lesssim \frac{\log \log R}{R^{n-1}}\|\mu\|_{R} .
$$

PROOF. In the proof of (3.4) we at no point used radiality of $\mu$.

In particular, taking $\nu=0$, we have that for arbitrary Borel measures $\mu$,

$$
\int_{|x|<1} \frac{d \mu(x)}{|x|^{n-1}} \lesssim \log \log R\|\mu\| \|_{R}
$$


REMARK. (1) In retrospect, the conjecture that (1.2) might hold was a little hasty. It was generated only by the single family of examples described, and we did not even take the effect of the tails of the expressions $|\widehat{g d \sigma}(R x)|$ into account. Considering the case $\delta \sim 1$, we should therefore have included 'tail' terms such as

$$
\sup _{y} \int_{1 / R \leq|x-y| \leq 1} \frac{d \mu(x)}{|x-y|^{n-1}}
$$

into consideration of necessary conditions. The fact is that, by the remark following the above corollary, and translation invariance of $\|\cdot \cdot\|_{R}$, this term (3.5) is automatically controlled by $\|\mu\| \|_{R}$ up to a factor of $\log \log R$. When $\mu$ is radial, the tails occurring in the (unmodulated, that is, $y=0$ ) cases $\delta \ll 1$ are controlled by the same mechanism, using explicit rotation invariance. However, when $y \neq 0$, and $\delta \ll 1$, it is perhaps simplest to use the theorem itself to see that the contributions from the tails (analogous to (3.5)) are dominated by $\log \log R\|\| \mu \|_{R}$.

In the case of general measures, it is easy to see that all of the 'tail' terms such as (3.5) are dominated by $\log R\|\| \mu \|_{R}$. (For (3.5) one uses a dyadic decomposition of $\{1 / R \leq|x-y| \leq 1\}$ and then tubes pointing along rays through $y$.) However, as we have noted above, (1.2) fails polynomially in $R$ for general measures $\mu$.

(2) If the Mizohata-Takeuchi conjecture 1.1 is correct, it can be rescaled to obtain the scale-invariant inequality

$$
\int_{\mathbb{R}^{n}}|\widehat{g d \sigma}(x)|^{2} d \mu \leq C\|\| \mu\|\| \|_{L^{2}\left(\mathbb{S}^{n-1}\right)}^{2}
$$

for all $g \in L^{2}\left(\mathbb{S}^{n-1}\right)$. (There is now no condition on the support of $\mu$.) However, it is not necessary that $\|\mu\| \|$ be finite in order to have

$$
\int_{\mathbb{R}^{n}}|\widehat{g d \sigma}(x)|^{2} d \mu \leq K(\mu)\|g\|_{L^{2}\left(\mathbb{S}^{n-1}\right)}^{2} .
$$

Indeed, taking $d \mu(x)=w(x) d x$, inequality (3.6) holds with $K(\mu)=\|w\|_{(n+1) / 2}$; this is the content of the Stein-Tomas restriction theorem. Of course, the quantities $\|w\|_{(n+1) / 2}$ and $\|\mu\| \|$ are not comparable. On the other hand, testing on the usual examples gives the necessary condition

$$
\sup \left\{\frac{\mu(T)}{N^{n-1}}: T \text { an } N \times N \cdots \times N \times N^{2} \text { tube, } N \geq 1\right\}<\infty
$$

for (3.6) to hold. The quantity \|\|$\mu \|$ is the smallest dilation-invariant functional of $\mu$ which is larger than that generated by (3.7). By Hölder's inequality, the left-hand side of (3.7) is at most $\|w\|_{(n+1) / 2}$ when $d \mu(x)=w(x) d x$. On the other hand, (3.7) is not sufficient for (3.6) to hold, as the result of the present paper shows. 


\section{Remarks on localised inequalities for general measures in two dimensions}

There are certain possible variants of inequality (1.2), and here we consider two such (the forthcoming inequalities (4.1) and (4.2)), neither one of which implies the other. Both originate in work on the Falconer distance set conjecture (see [8]). We omit the proofs due to their close proximity to arguments that are already well established in the literature.

4.1. A variant based on an argument of Erdoğan A careful inspection of the arguments in [6] (see also [5]) leads to the following two-weighted inequality for the extension operator in two dimensions. The subsequent variant of inequality (1.2) follows as a corollary.

In what follows $T(\alpha, \beta)$ will denote a rectangle in the plane of short side $\alpha$ and long side $\beta$, and $T_{\omega}(x ; \alpha, \beta)$ will be used to denote the rectangle $T(\alpha, \beta)$ centred at $x \in \mathbb{R}^{2}$ and pointing in the direction $\omega \in \mathbb{S}^{1}$. Here $\mathbb{B}$ denotes the unit ball in $\mathbb{R}^{2}$, and $\sigma$ the induced Lebesgue measure on the unit circle $\mathbb{S}^{1} \subset \mathbb{R}^{2}$.

THEOREM 4.1. Let $\mu$ be a Borel measure supported in $\mathbb{B}$. Then there exists an absolute constant $C$ such that for all $R \geq 1$ the a priori inequality

$$
\int_{\mathbb{B}}|\widehat{g d \sigma}(R \xi)|^{2} d \mu \leq C \frac{\log R}{R} \int_{\mathbb{S}^{1}}|g(\omega)|^{2} \mathcal{M}_{R} \mu(\omega) d \sigma(\omega)
$$

holds. Here the maximal function $\mathcal{M}_{R}$ is given by

$$
\mathcal{M}_{R} \mu(\omega)=\sup _{R^{-1} \leq \alpha \leq R^{-1 / 2}} \sup _{\substack{T|| \omega \\ T=T\left((\alpha R)^{-1}, 1\right)}}\left(\frac{1}{|T|} \int_{T}\left(\frac{\mu\left(T_{\omega}\left(x ; \alpha, \alpha^{2} R\right)\right)}{\left|T_{\omega}\right|}\right)^{2} d x\right)^{1 / 2} .
$$

COROLlaRY 4.2. There exists a constant $C$ such that

$$
\int_{\mathbb{B}}|\widehat{g d \sigma}(R \xi)|^{2} d \mu \leq C \frac{\log R}{R} \sup _{R^{-1} \leq \alpha \leq R^{-1 / 2}} \sup _{\substack{T=T\left(\alpha, \alpha^{2} R\right) \\ T^{\prime}=T^{\prime}\left((\alpha R)^{-1}, 1\right) \\ T \subset T^{\prime}, T \| T^{\prime}}}\left\{\frac{\mu(T) \mu\left(T^{\prime}\right)}{\left|T \| T^{\prime}\right|}\right\}^{1 / 2}\|g\|_{2}^{2},
$$

for all Borel measures $\mu$ supported in the unit ball, $R \geq 1$ and $g \in L^{2}\left(\mathbb{S}^{n-1}\right)$.

4.2. A variant based on an argument of Bourgain A careful inspection of the arguments in [3] leads to the following variant of inequality (1.2). This observation is due to Ana Vargas [11].

THEOREM 4.3. There exists a constant $C$ such that

$$
\int_{\mathbb{B}}|\widehat{g d \sigma}(R \xi)|^{2} d \mu \leq C \frac{(\log R)^{1 / 3}}{R^{5 / 6}} \sup _{T=T\left(R^{-1 / 2}, 1\right)}\left\{\frac{\mu(T)}{|T|}\right\}^{1 / 3}\|\mu\|_{2}^{2 / 3}\|g\|_{2}^{2},
$$

for all Borel measures $\mu$ supported in the unit ball, $R \geq 1$ and $g \in L^{2}\left(\mathbb{S}^{n-1}\right)$. 


\section{References}

[1] J. A. Barceló, J. M. Bennett, A. Carbery, A. Ruiz and M. C. Vilela, 'Some special solutions to the Schrödinger equation', Indiana Univ. Math. J. 56(4) (2007), 1581-1593.

[2] J. A. Barceló, A. Ruiz and L. Vega, 'Weighted estimates for the Helmholtz equation and some applications', J. Funct. Anal. 150(2) (1997), 356-382.

[3] J. Bourgain, 'Hausdorff dimension and distance sets', Israel J. Math. 87 (1994), 193-201.

[4] A. Carbery and F. Soria, 'Pointwise Fourier inversion and localisation in $\mathbb{R}^{n}$ ', J. Fourier Anal. Appl. 3 (1997), 847-858 (special issue).

[5] A. Carbery and A. Seeger, 'Weighted inequalities for Bochner-Riesz means in the plane', $Q . J$. Math. 51 (2000), 155-167.

[6] M. B. Erdoğan, 'A note on the Fourier transform of fractal measures', Math. Res. Lett. 11(2-3) (2004), 299-313.

[7] A. Iosevich and M. Rudnev, 'Distance measures for well-distributed sets', Discrete Comput. Geom. 38(1) (2007), 61-80.

[8] P. Mattila, Geometry of Sets and Measures in Euclidean Spaces, Cambridge Studies in Advanced Mathematics, 44 (Cambridge University Press, Cambridge, 1995).

[9] S. Mizohata, On the Cauchy Problem, Notes and Reports in Mathematics, Science and Engineering, 3 (Academic Press, San Diego, CA, 1985).

[10] E. M. Stein, Harmonic Analysis (Princeton University Press, Princeton, NJ, 1993).

[11] A. Vargas, Personal communication.

[12] G. N. Watson, The Theory of Bessel Functions (Cambridge University Press, Cambridge, 1969).

[13] T. H. Wolff, 'Decay of circular means of Fourier transforms of measures', Internat. Math. Research Notices 10 (1999), 547-567.

J. A. BARCELÓ, ETSI de Caminos, Universidad Politécnica de Madrid, 28040, Madrid, Spain

e-mail: juanantonio.barcelo@upm.es

J. M. BENNETT, School of Mathematics, The Watson Building, University of Birmingham, Edgbaston, Birmingham, B15 2TT, UK e-mail: J.Bennett@bham.ac.uk

A. CARBERY, School of Mathematics and Maxwell Institute for Mathematical Sciences, University of Edinburgh, JCMB, King's Buildings, Mayfield Road, Edinburgh, EH9 3JZ, UK

e-mail: A.Carbery@ed.ac.uk 